\title{
Starburst activity in the host galaxy of the $z=2.58$ quasar J1409+5628 ${ }^{\star}$
}

\author{
A. Beelen ${ }^{1}$, P. Cox ${ }^{1}$, J. Pety ${ }^{2}$, C. L. Carilli ${ }^{3}$, F. Bertoldi ${ }^{4}$, E. Momjian ${ }^{5}$, A. Omont ${ }^{6}$, \\ P. Petitjean ${ }^{6}$, and A. O. Petric ${ }^{7}$
}

${ }^{1}$ Institut d'Astrophysique Spatiale, Université de Paris XI, 91405 Orsay, France e-mail: Alexandre.Beelen@ias.u-psud.fr

2 IRAM, 300 rue de la Piscine, 38406 St-Martin-d'Hères, France

3 National Radio Astronomy Observatory, PO Box, Socorro, NM 87801, USA

4 Max-Planck-Institut für Radioastronomie, Auf dem Hügel 69, 53121 Bonn, Germany

5 NAIC, Arecibo Observatory, HC 3 Box 53995, Arecibo, PR 00612, USA

${ }^{6}$ Institut d'Astrophysique de Paris, CNRS \& Université Paris 6, 98 bis Bd. Arago, 75014 Paris, France

7 Astronomy Department, Columbia University, New York, NY USA

Received 23 February 2004 / Accepted 30 April 2004

\begin{abstract}
We report the detection of CO emission from the optically luminous, radio-quiet quasar J140955.5+562827 (hereafter $\mathrm{J} 1409+5628)$, at a redshift $z_{\mathrm{CO}}=2.583$. We also present VLA continuum maps and VLBA high spatial resolution observations at $1.4 \mathrm{GHz}$. Both the $\mathrm{CO}(3 \rightarrow 2)$ and $\mathrm{CO}(7 \rightarrow 6)$ emission lines are detected using the IRAM Plateau de Bure interferometer. The $3 \rightarrow 2 / 7 \rightarrow 6$ line luminosity ratio is about $1 / 3$, indicating the presence of warm and dense molecular gas with an estimated mass of $6 \times 10^{10} M_{\odot}$. The infrared-to-CO luminosity ratio $L_{\mathrm{FIR}} / L_{\mathrm{CO}(1 \rightarrow 0)}^{\prime} \approx 500 L_{\odot}\left(\mathrm{K} \mathrm{km} \mathrm{s}^{-1} \mathrm{pc}^{2}\right)^{-1}$, comparable to values found for other high- $z$ sources where CO line emission is seen. J1409+5628 is detected using the VLA with a $1.4 \mathrm{GHz}$ rest-frame luminosity density of $4.0 \times 10^{25} \mathrm{~W} \mathrm{~Hz}^{-1}$. The rest-frame radio to far-infrared ratio, $q$, has a value of 2.0 which is similar to the values found in star forming galaxies. At the 30 mas resolution of the VLBA, J1409+5628 is not detected with a $4 \sigma$ upper limit to the surface brightness of $0.29 \mathrm{mJy}_{\text {beam }}^{-1}$. This implies a limit to the intrinsic brightness temperature of $2 \times 10^{5} \mathrm{~K}$ at $8 \mathrm{GHz}$, typical for nuclear starbursts and more than two orders of magnitude fainter than typical radio-loud active galactic nuclei. Both the properties of the $\mathrm{CO}$ line emission and the radio emission from J1409+5628 are therefore consistent with those expected for a star forming galaxy. In J1409+5628 young massive stars are the dominant source of dust heating, accounting for most of the infrared luminosity. The massive reservoir of molecular gas can sustain the star formation rate of a few $1000 M_{\odot} \mathrm{yr}^{-1}$ implied by the far-infrared luminosity for about 10 million years.
\end{abstract}

Key words. Galaxy: formation - galaxies: starburst - galaxies: high-redshift - galaxies: quasars: emission lines galaxies: quasars: individual: J140955.5+562827 - cosmology: observations

\section{Introduction}

In the recent decade, millimeter and submillimeter deep blank field surveys (e.g., Ivison et al. 2000; Blain et al. 2002) and pointed observations (e.g., Omont et al. 2003; Priddey et al. 2003; Bertoldi et al. 2003a) have provided a view of the dust content of a few hundred galaxies and quasars in the redshift range $1<z<6.4$. In these objects, a significant fraction of the energy generated by star formation is processed by dust and re-emitted at far-infrared wavelengths. The average space density of ultraluminous infrared galaxies and quasars at high- $z$ is found to be thousandfold greater than in the local universe. Observations at millimeter and submillimeter wavelengths

* This paper is based on observations obtained with the IRAM Plateau de Bure Interferometer. IRAM is funded by Centre National de la Recherche Scientifique (France), the Max-Planck-Gesellschaft (Germany), and the Instituto Geografico Nacional (Spain). provide a direct way to trace the bulk of the star formation in the early universe.

Pointed submillimeter and millimeter observations of optically selected quasars or radio galaxies have the advantage over deep field surveys in providing unique source identification and redshifts. In particular, targeted observations of optically luminous quasars have been successful in revealing massive bursts of star formation in their host galaxies. Although optically luminous quasars, which trace the most massive collapsed structures to have formed in the early universe, are rare objects which bear little relation to the objects that make up most of the far-infrared and submillimeter background, they provide powerful probes to study the relation between star formation, massive gas reservoirs, and the growth of super-massive black holes in the dark ages of the Universe (Fan et al. 2003; Bertoldi et al. 2003b; Walter et al. 2003). 
The study of the redshift range $2<z<3$ is important in studying the star formation history in the universe since it traces the peak of the space density of the quasar population (Shaver et al. 1996), and corresponds to the median redshift of submillimeter galaxies (Chapman et al. 2003). Recent pointed observations of the 1.2 and $0.85 \mathrm{~mm}$ thermal dust continuum emission of $z \approx 2$ optically luminous, radio-quiet quasars reveal that $1 / 3$ of these quasars are also luminous in the infrared with far-infrared luminosities of $L_{\mathrm{FIR}} \sim 10^{13} L_{\odot}$ and estimated dust masses of typically $\sim 10^{8} M_{\odot}$ (Omont et al. 2003; Priddey et al. 2003).

The optically very bright $\left(M_{\mathrm{B}}=-28.4\right)$, radio-quiet quasar $\mathrm{J} 1409+5628$ is by far the strongest $\mathrm{mm}$ source in the Omont et al. (2003) survey. With an estimated far-infrared luminosity of $4 \times 10^{13} L_{\odot}, \mathrm{J} 1409+5628$ ranges amongst the most luminous infrared high- $z$ sources found to date. Under the assumption that the dust is predominantly heated by massive stars, the inferred star formation rate is several $1000 M_{\odot} \mathrm{yr}^{-1}$ (Omont et al. 2003). However, the fraction of infrared luminosity due to dust heated by star formation and dust directly heated by the Active Galactic Nuclei (AGN) in J1409+5628 is still an open issue, as is the case for almost all of the high- $z$ quasars studied to date. Both the large masses of dust and the high star formation rate indicate that $\mathrm{J} 1409+5628$ should have a copious reservoir of molecular gas to sustain the formation of a few $1000 M_{\odot}$ per year over its dynamical time.

In high- $z$ sources, the search for molecular gas is best done by using the redshifted rotational lines of CO. The molecular gas in star-forming galaxies is warm and dense enough to excite the higher $\mathrm{CO}$ rotational levels $(J>3)$ which are shifted into the millimeter windows for redshifts greater than 2 . However, a good determination of the redshift of the target object, either from optical or near-infrared spectroscopy, is a prerequisite for a detection because the bandwidths of the current heterodyne receivers are still relatively narrow. For example, at the Plateau de Bure Interferometer, the available instantaneous bandwitdh is $\Delta v=580 \mathrm{MHz}$ which corresponds at $96 \mathrm{GHz}$ to a velocity range of $1810 \mathrm{~km} \mathrm{~s}^{-1}$ or $\Delta z \sim 0.02$ at $z=2.6$.

Despite this current limitation, over the last few years CO line emission has been detected in 24 far-infrared luminous high- $z$ quasars, radio and (sub)mm galaxies (Carilli et al. 2004, and references therein). These observations reveal large reservoirs of molecular gas $\left(10^{11} M_{\odot}\right)$, a prerequisite for efficient star formation, and prompted also the first studies on the properties of the dense interstellar gas in starburst galaxies in the early universe (e.g., Barvainis et al. 1997; Solomon et al. 2003; Bertoldi et al. 2003b). In addition, these observations allow us to estimate, unhindered by extinction, the dynamical masses in these systems, which are the key constraining hierarchical models (see, e.g., Neri et al. 2003).

In this paper, we present a study of the $\mathrm{CO}$ emission in $\mathrm{J} 1409+5628$ made with the Plateau de Bure interferometer together with observations of the radio emission carried out with the Very Large Array and the Very Large Baseline Array. The data provide strong evidence that the origin of the infrared luminosity is dominated by the starburst activity of the host galaxy of the QSO J1409+5628. Throughout this paper, we adopt the concordance $\Lambda$-cosmology with
$H_{0}=71 \mathrm{~km} \mathrm{~s}^{-1} \mathrm{Mpc}^{-1}, \Omega_{\Lambda}=0.73$ and $\Omega_{\mathrm{m}}=0.27-($ Spergel et al. 2003). At $z=2.58$, the luminosity distance $D_{L}$ is $21.5 \mathrm{Gpc}$ and an angular scale of $1^{\prime \prime}$ corresponds to $8.1 \mathrm{kpc}$.

\section{Observations}

\subsection{Plateau de Bure interferometer}

Observations were made with the IRAM Plateau de Bure interferometer in May and June 2002 and from January to March 2003. We used the D and B configurations, as well as non-standard configurations, with 4 to 6 antennas, resulting in a good coverage of the $u v$ plane with baselines ranging from 24 to $400 \mathrm{~m}$. This results in final synthesized beams of 2 .' $44 \times$ $1^{\prime \prime} .65$ at a position angle of $60^{\circ}$ at $3.1 \mathrm{~mm}$, and $1.01^{\prime \prime} \times 0.55^{\prime \prime}$ with a position angle of $52^{\circ}$ at $1.3 \mathrm{~mm}$.

The dual frequency set-up was used to search simultaneously for the $\mathrm{CO}(3 \rightarrow 2)$ emission line and dust emission at 3 and $1.3 \mathrm{~mm}$, and subsequently for the $\mathrm{CO}(7 \rightarrow 6)$ emission line. During the first series of observations, the $3.1 \mathrm{~mm}$ receiver was tuned to a central frequency of $96.780 \mathrm{GHz}$, corresponding to the $\mathrm{CO}(3 \rightarrow 2)$ line at a redshift of $z=2.5730$, close to the strong Lyman- $\alpha$ absorption line seen at $z=2.5758$ in the optical spectrum of $\mathrm{J} 1409+5628$ (see Sect. 4.1). The $\mathrm{CO}(3 \rightarrow 2)$ line emission was tentatively detected at the low frequency end of the available bandwidth. In subsequent observations, we retuned the $3.1 \mathrm{~mm}$ receivers to $96.505 \mathrm{GHz}$, corresponding to a redshift of $z=2.583$ and confirmed this tentative detection. After the first tentative detection of the $\mathrm{CO}(3 \rightarrow 2)$ emission line, we retuned the $1.3 \mathrm{~mm}$ receivers in the upper sideband to $225.198 \mathrm{GHz}$ corresponding to the redshifted $\mathrm{CO}(7 \rightarrow 6)$ emission line, while the lower sideband was tuned to a frequency $3 \mathrm{GHz}$ away (at $225 \mathrm{GHz}$ ) to measure the $1.3 \mathrm{~mm}$ continuum. Typical SSB system temperatures were $\approx 150 \mathrm{~K}$ and $\approx 400 \mathrm{~K}$ at $3 \mathrm{~mm}$ and $1.3 \mathrm{~mm}$ respectively.

The data were reduced, calibrated and analyzed using the IRAM package GILDAs. During the data reduction, we flagged antennas with too low efficiencies or too high system temperatures, and discarded data taken during bad atmospheric conditions. The final usable datasets correspond to total on-source integration times of 52 and $27 \mathrm{~h}$ at 3.1 and $1.3 \mathrm{~mm}$, respectively. After phase and amplitude calibration of each session, we combined the data from the different settings into one single table at full spectral resolution. The flux calibration was done using standard calibrators 3C 273, CRL 618 or MWC 349.

\subsection{Very large array}

J1409+5628 was observed with the Very Large Array (VLA) of the $\mathrm{NRAO}^{1}$ at $1.4 \mathrm{GHz}$ in the A configuration (maximum baseline of $30 \mathrm{~km}$ ), with a total bandwidth of $100 \mathrm{MHz}$ and two polarizations. The total observing time was $2 \mathrm{~h}$. The entire dataset was self-calibrated using field sources, and standard phase and amplitude calibration was applied for J1409+5628.

1 The National Radio Astronomy Observatory is a facility of the National Science Foundation operated under cooperative agreement by Associated Universities, Inc. 

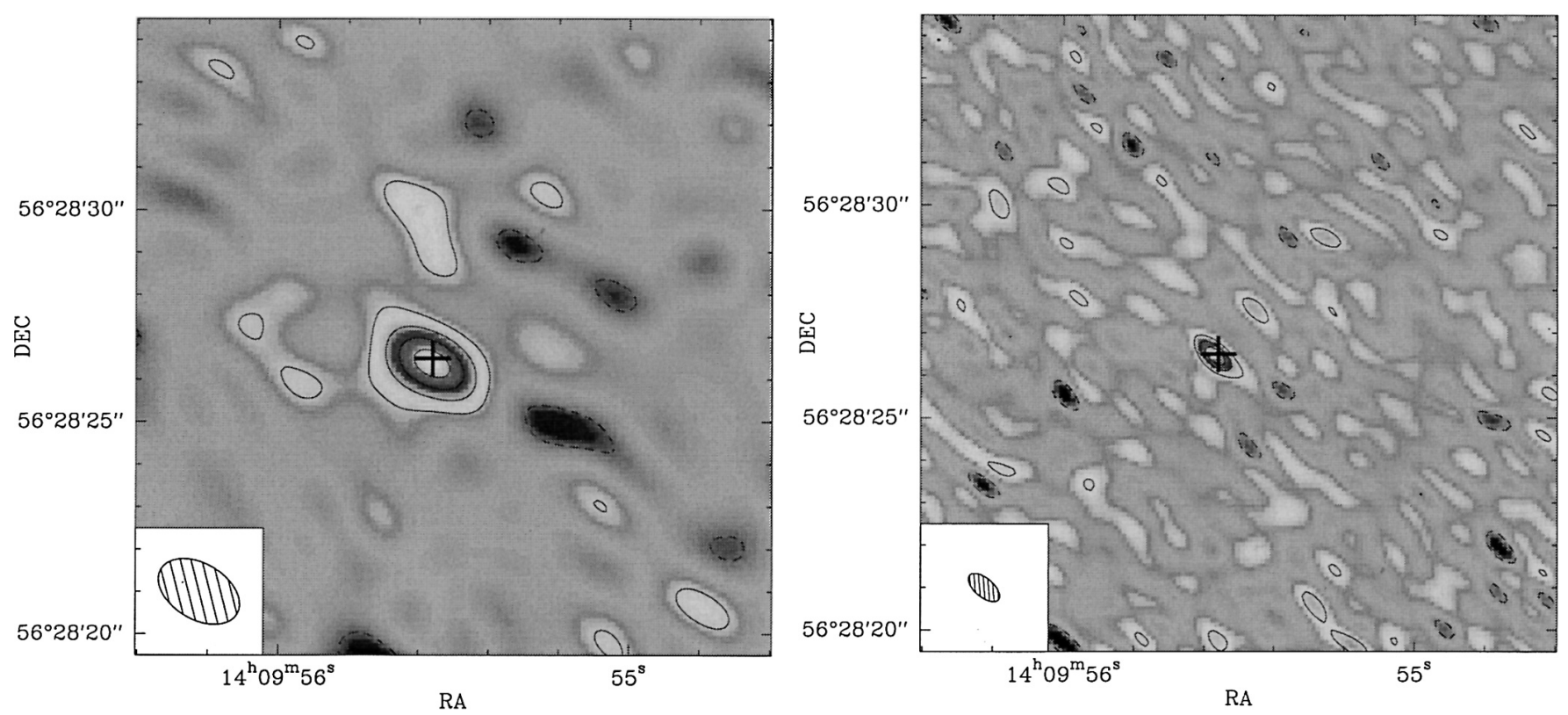

Fig. 1. Left panel: velocity-integrated map of the $\mathrm{CO}(3 \rightarrow 2)$ line emission toward J $1409+5628$. The contour step is $0.3 \mathrm{mJy} / \mathrm{beam}$, corresponding to $2 \sigma$, dashed contours are negative. Right panel: velocity-integrated map of the $\mathrm{CO}(7 \rightarrow 6)$ line and $1.3 \mathrm{~mm}$ continuum emission toward $\mathrm{J} 1409+5628$. The contour step is $2 \mathrm{mJy} /$ beam, corresponding to $2 \sigma$, dashed contours are negative. In both panels, the cross shows the optical position of the quasar taken from the 2MASS catalogue. The synthesized beams are shown in the lower left corner of each panel.

The absolute flux density scale was set with the observations of $3 \mathrm{C} 286$.

The final image was generated using the wide field imaging and deconvolution capabilities of the NRAO's Astronomical Image Processing System (AIPS) task IMAGR. The present $1.4 \mathrm{GHz}$ continuum data achieved a rms noise $(\sigma)$ of $16 \mu \mathrm{Jy}$ which corresponds to the theoretical expected noise level. The Gaussian restoring CLEAN beam full width at half-maximum $(F W H M)$ is $\sim 1$ '.5. A detailed discussion of the VLA data will be given in Petric et al. (in preparation).

\subsection{Very long baseline array}

We observed J1409+5628 with the VLBA of the NRAO on the 9th of March and 7th of April, 2003, for a total of $14 \mathrm{~h}$ using the standard continuum mode at $1.4 \mathrm{GHz}$ (total bandwidth of $16 \mathrm{MHz}$ in two dual circular polarizations). The calibrator J1408+5613 was used for phase referencing with a calibration cycle time of $3.5 \mathrm{~min}$. Standard a priori gain calibration was applied. At $1.4 \mathrm{GHz}$, the VLBA short spacing limit filters out all spatial structure larger than about 0 .' 15 .

A number of test cycles were also included to monitor the coherence of the phase referencing. These tests involved switching between two calibrators (J1408+5613 and $\mathrm{J} 1419+5423$ ) with a similar angular separation and cycle time as that used for the target source. Images of the second calibrator (J1419+5423) were deconvolved using two different approaches: by applying the phase and amplitude selfcalibration solutions of the phase reference source J1408+5613 on $\mathrm{J} 1419+5423$, and by self calibrating $\mathrm{J} 1419+5423$ itself, both in phase and amplitude. The ratio of the peak surface brightness between the final images of the two approaches gives a measure of the effect of residual phase errors after phase referencing (i.e. "the coherence" due to phase referencing). At all times the coherence was found to be better than 95\%. Also, phase referencing as used herein is known to preserve absolute astrometric positions to better than 0.' 1 (Fomalont 1999).

\section{Results}

\subsection{Molecular gas}

The velocity-integrated maps of the $\mathrm{CO}(3 \rightarrow 2)$ and $\mathrm{CO}(7 \rightarrow 6)$ emission lines of $\mathrm{J} 1409+5628$ are shown in Fig. 1 and the corresponding spectra are displayed in Fig. 2. The $\mathrm{CO}(3 \rightarrow 2)$ line is detected with high signal-to-noise ratio at a central frequency of $96.504 \mathrm{GHz}$, corresponding to a redshift $z_{\mathrm{CO}}=2.5832 \pm 0.0001$. At the same redshift, the $\mathrm{CO}(7 \rightarrow 6)$ emission line is also detected on top of the $1.3 \mathrm{~mm}$ continuum.

Within the astrometric uncertainties, the $\mathrm{CO}$ emission and the $1.3 \mathrm{~mm}$ continuum, centered at $14^{\mathrm{h}} 09^{\mathrm{m}} 55^{\mathrm{s}} .5+56^{\circ} 28^{\prime} 26^{\prime \prime} .4$ (J2000.0), coincide with the optical position listed in the 2MASS catalogue (Barkhouse \& Hall 2001). At the $1.0^{\prime \prime} \times$ $0.5^{\prime \prime}$ resolution of our Plateau de Bure observations at $1.3 \mathrm{~mm}$, the $\mathrm{CO}(7 \rightarrow 6)$ emission in $\mathrm{J} 1409+5628$ is still unresolved, yielding an upper limit to the linear size of the region emitting in $\mathrm{CO}$ of $5 \mathrm{kpc}$.

We fitted the high signal-to-noise $\mathrm{CO}(3 \rightarrow 2)$ spectrum with a Gaussian profile where the central frequency $\left(v_{\mathrm{obs}}\right)$, the full width half maximum $\left(\Delta v_{F W H M}\right)$, the continuum flux density $\left(S_{v}\right)$, and the integrated $\mathrm{CO}$ emission flux density $\left(I_{\mathrm{CO}}\right)$ were kept as free parameters. The peak intensity was derived from the Gaussian fit parameters. The best Gaussian fit yields a line width to the $\mathrm{CO}(3 \rightarrow 2)$ emission of $311 \pm$ $28 \mathrm{~km} \mathrm{~s}^{-1}$, similar to the widths found in other high- $z$ quasars 


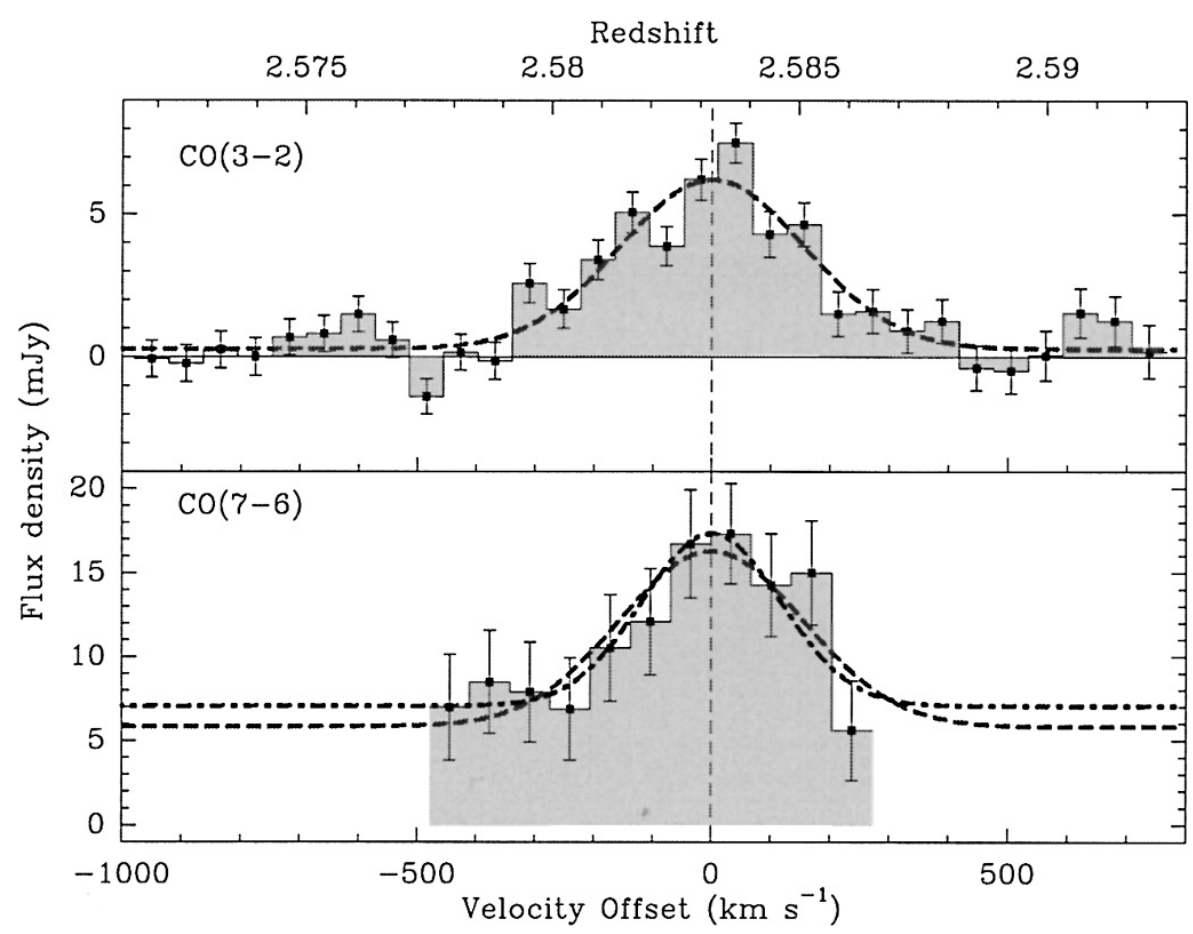

Fig. 2. Observed spectra of the $\mathrm{CO}(3 \rightarrow 2)$ and $\mathrm{CO}(7 \rightarrow 6)$ line emission toward $\mathrm{J} 1409+5628$, with velocity resolutions of 58 and $76 \mathrm{~km} \mathrm{~s}^{-1}$, respectively. The offset velocity corresponds to central frequencies of 96.504 and $225.1197 \mathrm{GHz}$. The error bars represent the statistical uncertainties from the fit in the $u v$-plane. The dashed lines show the Gaussian fits together with the fit to the continuum with the parameters listed in Table 1. The dot-dashed line is the fit to the $\mathrm{CO}(7 \rightarrow 6)$ line emission leaving the linewidth as a free parameter. (e.g., Cox et al. 2002), and a peak intensity of $6 \pm 1 \mathrm{mJy}$. The velocity-integrated $\mathrm{CO}(3 \rightarrow 2)$ flux is $2.3 \pm 0.2 \mathrm{Jy} \mathrm{km} \mathrm{s}^{-1}$. At the position of $\mathrm{J} 1409+5628$ we obtain from the combined fit a $3 \sigma$ upper limit to the continuum flux density at $3.1 \mathrm{~mm}$ of $S_{96.5 \mathrm{GHz}}<0.5 \mathrm{mJy}$.

The width and centroid of the Gaussian fit to the $\mathrm{CO}(3 \rightarrow 2)$ line were adopted for the profile of the lower signalto-noise ratio $\mathrm{CO}(7 \rightarrow 6)$ emission line. The peak intensity of the $\operatorname{CO}(7 \rightarrow 6)$ emission is $10 \pm 3 \mathrm{mJy}$, the velocity-integrated $\mathrm{CO}(7 \rightarrow 6)$ flux $4.1 \pm 1.0 \mathrm{Jy} \mathrm{km} \mathrm{s}^{-1}$, and the $1.3 \mathrm{~mm}$ continuum is detected at a flux density of $6 \pm 2 \mathrm{mJy}$. The $\mathrm{CO}(7 \rightarrow 6)$ emission line is detected with a signal-to-noise ratio of 4 , as compared to the ratio of 11 in the case of $\mathrm{CO}(3 \rightarrow 2)$. The $\mathrm{CO}(7 \rightarrow 6)$ line flux is therefore somewhat uncertain, and the precise value depends on how the spectrum is analyzed. For instance, leaving the line width as a free parameter in the Gaussian fitting yields $\Delta v_{F W H M}=229 \pm 84 \mathrm{~km} \mathrm{~s}^{-1}$ and a $\mathrm{CO}(7 \rightarrow 6)$ integrated line flux of $3.0 \pm 1.3 \mathrm{Jy} \mathrm{km} \mathrm{s}^{-1}$, i.e. a $2.3 \sigma$ result (Fig. 2).

The upper limit of the continuum flux density at $96 \mathrm{GHz}$ and the continuum flux density at $225 \mathrm{GHz}$ are consistent with the $250 \mathrm{GHz}$ flux density of $S_{250 \mathrm{GHz}}=10.7 \pm 0.6 \mathrm{mJy}$ (Omont et al. 2003), for a grey-body spectrum with a dust temperature of $45 \mathrm{~K}$ and a spectral index $\beta=1.5$. The corresponding far-infrared luminosity is $L_{\mathrm{FIR}} \approx 4.3 \times 10^{13} L_{\odot}$. The recent detection of $\mathrm{J} 1409+5628$ at $350 \mu \mathrm{m}$ is in agreement with these conclusions (Beelen et al., in preparation).

\subsection{Non-thermal radio emission}

Figures 3 and 4 show the VLA and VLBA radio images of $\mathrm{J} 1409+5628$ at $1.4 \mathrm{GHz}$. The VLA image at 1".6 resolution shows an unresolved source with a deconvolved size $<0.5$, consistent with the upper limit derived from

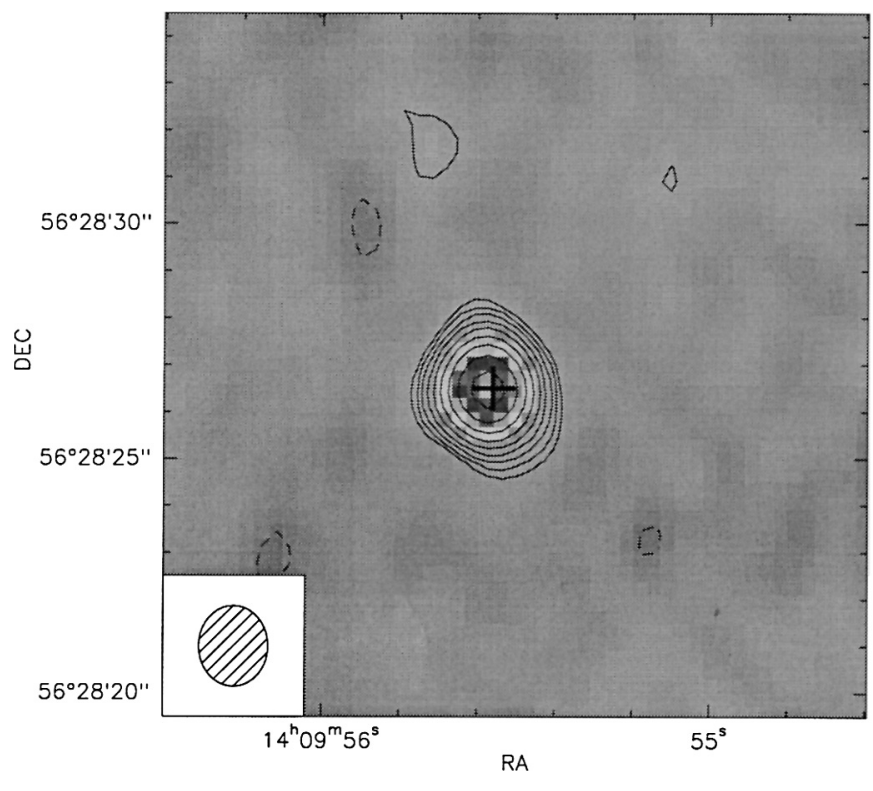

Fig. 3. VLA image of $\mathrm{J} 1409+5628$ at $1.4 \mathrm{GHz}$ at a resolution of 1 .'7 $\times$ $1^{\prime \prime} 5(F W H M)$, major axis position angle of $5^{\circ}$. The contours are a geometric progression in the square root of two, with the first contour level at $0.05 \mathrm{mJy} / \mathrm{beam}$, and the highest one at $128 \sigma$. Negative contours are dashed.

the $\mathrm{CO}$ measurements. The flux density at $1.4 \mathrm{GHz}$ is $S_{1.4 \mathrm{GHz}}=0.93 \pm 0.022 \mathrm{mJy}$. The radio source position is $14^{\mathrm{h}} 09^{\mathrm{m}} 55^{\mathrm{s}} .57+56^{\circ} 28^{\prime} 26^{\prime \prime} \cdot 47$ (J2000), and coincides within the astrometric errors $(\leq 0.2)$ with the positions of the optical and the $\mathrm{CO}$ emission.

The VLBA image at full resolution $(12.5 \mathrm{mas} \times 6.7 \mathrm{mas}$, major axis position angle $-9^{\circ}$; left panel of Fig. 4) has an rms noise of $46 \mu \mathrm{Jy}$ beam $^{-1}$. There is no source brighter than $4 \sigma$ in a field of 0.5 centered on the VLA position. We have also 

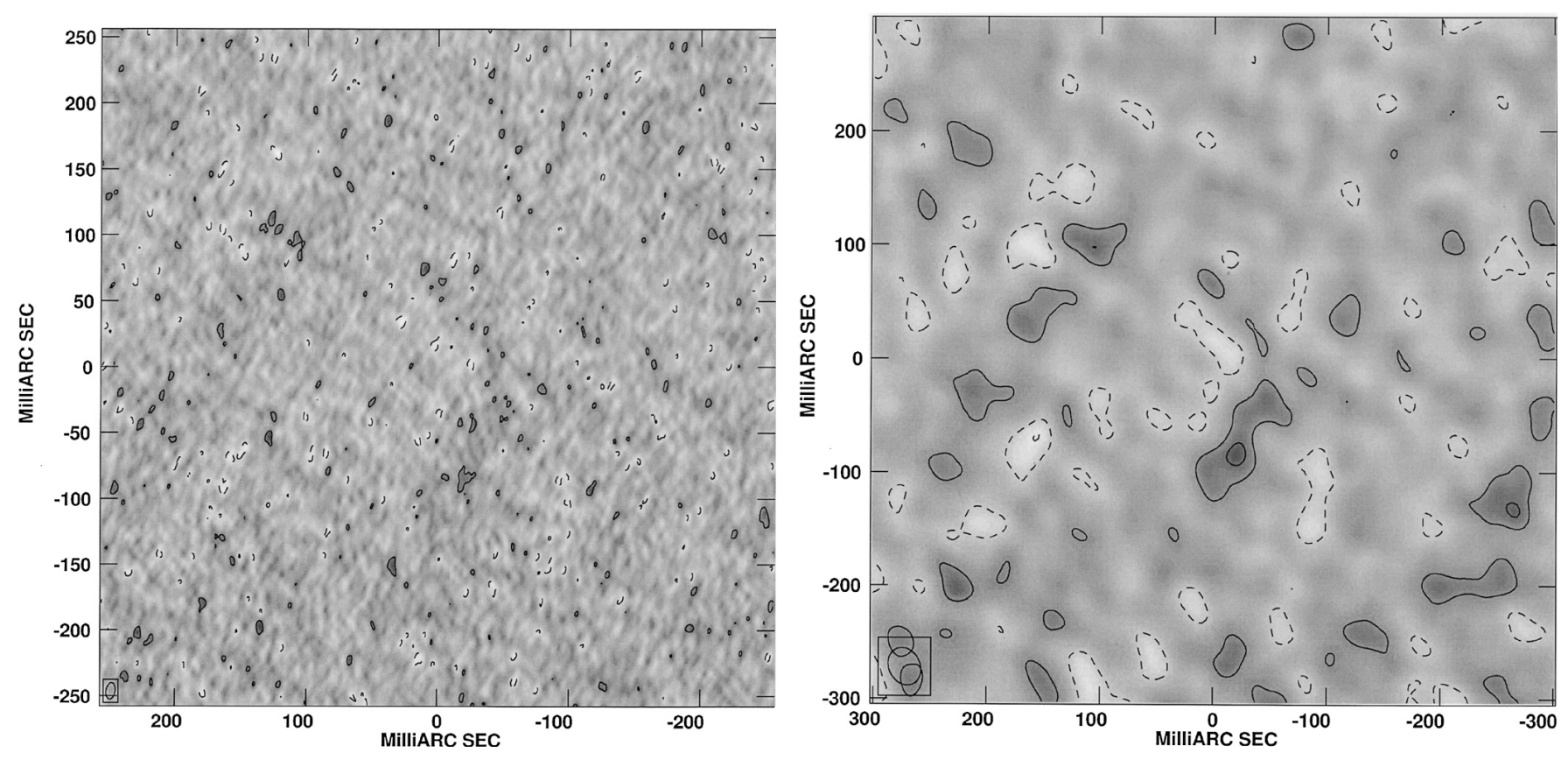

Fig. 4. Left panel: VLBA $1.4 \mathrm{GHz}$ image at full resolution $\left(12.5 \mathrm{mas} \times 6.7\right.$ mas, major axis position angle $\left.-9^{\circ}\right)$. The contours levels are: -0.3 , $-0.2,-0.1,0.1,0.2,0.3,0.4 \mathrm{mJy}_{\text {beam }}^{-1}$. Right panel: the VLBA $1.4 \mathrm{GHz}$ image tapered to $34 \times 28$ mas resolution. The contours are: -0.33 , $-0.22,-0.11,0.11,0.22,0.33,0.44 \mathrm{mJy}_{\text {beam }}{ }^{-1}$. The central position corresponds to $14^{\mathrm{h}} 09^{\mathrm{m}} 55^{\mathrm{s}} .5739,+56^{\circ} 28^{\prime} 26^{\prime \prime} 475$ (J2000).

Table 1. Properties of the CO lines observed toward J1409+5628.

\begin{tabular}{ccccccccc}
\hline \hline Line & $\begin{array}{c}v_{\text {obs }} \\
{[\mathrm{GHz}]}\end{array}$ & $z_{\mathrm{CO}}$ & $\begin{array}{c}\text { Peak int. } \\
{[\mathrm{mJy}]}\end{array}$ & $\begin{array}{c}\Delta v_{F W H M} \\
{\left[\mathrm{~km} \mathrm{~s}^{-1}\right]}\end{array}$ & $\begin{array}{c}S_{v} \\
{[\mathrm{mJy}]}\end{array}$ & $\begin{array}{c}I_{\mathrm{CO}} \\
{\left[\mathrm{Jy} \mathrm{km} \mathrm{s}^{-1}\right]}\end{array}$ & $\begin{array}{c}L_{\mathrm{CO}}^{\prime} \\
{\left[10^{10} \mathrm{~K} \mathrm{~km} \mathrm{~s}^{-1} \mathrm{pc}^{2}\right]}\end{array}$ & $\begin{array}{c}L_{\mathrm{CO}} \\
{\left[10^{8} L_{\odot}\right]}\end{array}$ \\
\hline $\mathrm{CO}(3 \rightarrow 2)$ & 96.504 & $2.5832 \pm 0.0001$ & $6 \pm 1$ & $311 \pm 28$ & $<0.5^{\dagger}$ & $2.3 \pm 0.2$ & $8.2 \pm 0.6$ & $1.1 \pm 0.1$ \\
$\mathrm{CO}(7 \rightarrow 6)$ & 225.1197 & $\diamond$ & $10 \pm 3$ & $\diamond$ & $6 \pm 2$ & $4.1 \pm 1.0$ & $2.6 \pm 0.7$ & $4.4 \pm 1.2$ \\
\hline
\end{tabular}

Notes: ${ }^{\dagger} 3 \sigma$ level upper limit. ${ }^{\diamond}$ Adapting the $\mathrm{CO}(3 \rightarrow 2)$ parameters (see text).

made tapered images at lower resolution and wider fields. At $34 \times 28$ mas resolution the rms noise is $72 \mu \mathrm{Jy} \mathrm{beam}^{-1}$. The tapered image is shown in the right panel of Fig. 4. We still find no source brighter than $4 \sigma$ within 0.3 of the field center. Near the center of the field there is a "linear" feature extending about 0. ' 1 north-south with a peak surface brightness of $0.27 \pm$ $0.07 \mathrm{mJy}$ beam $^{-1}$ at $14^{\mathrm{h}} 09^{\mathrm{m}} 55^{\mathrm{s}} .572,+56^{\circ} 28^{\prime} 26^{\prime \prime} .39$ (J2000). It is possible that this linear feature corresponds to the $\mathrm{J} 1409+5628$ radio source, but given the low surface brightness, we conclude that our VLBA observations can only set a $4 \sigma$ upper limit to the surface brightness at 30 mas resolution of $0.29 \mathrm{mJy} \mathrm{beam}^{-1}$.

\section{Discussion}

\subsection{The redshift of $\mathrm{J} 1409+5628$}

The redshift derived from the $\mathrm{CO}$ measurements should correspond to the systemic redshift of the host galaxy of $\mathrm{J} 1409+5628$, since it traces the extended molecular gas associated with the quasar and not ionized gas undergoing energetic processes linked with the AGN activity. The derived redshift for the molecular gas, $z_{\mathrm{CO}}=2.5832 \pm 0.0001$, is in the upper part of the redshift range determined from the optical spectra (Korista et al. 1993; Barlow \& Junkkarinen 1994), i.e. $2.550<z<2.58$. $z_{\mathrm{CO}}$ is close to the redshift of the strong Ly- $\alpha$ absorption $(z=2.5758)$ seen in the Keck spectrum (Korista et al. 1993). This absorption line is associated with narrow $\mathrm{C}$ IV absorption at $4352 \AA$, indicating that the corresponding gas is not part of the Lyman- $\alpha$ forest, but rather part of the host-galaxy. This is in agreement with the fact that this ionization state is not associated with the central AGN (see, e.g., Petitjean et al. 1994) and that its redshift is close to $z_{\mathrm{CO}}$. The (systemic) velocity corresponding to $z_{\mathrm{CO}}$ is redshifted by $1800 \mathrm{~km} \mathrm{~s}^{-1}$ from the $z=2.5624$ high ionization ultraviolet emission lines such as C Iv (Korista et al. 1993), a difference which has been found in many other quasars (Richards et al. 2002).

\subsection{Molecular gas emission}

The $L_{\mathrm{CO}(7 \rightarrow 6)}^{\prime}$ luminosity is about $1 / 3$ of the $L_{\mathrm{CO}(3 \rightarrow 2)}^{\prime}$ luminosity (Table 1 ), a ratio which is comparable within a factor of 2 to the $7 \rightarrow 6 / 3 \rightarrow 2$ luminosity ratios measured in the high- $z$ sources SMM J14011+0252 (0.2, Downes \& Solomon 2003), H1413+117, i.e. the Cloverleaf (0.8, Barvainis et al. 1997), and J1148+5251 (0.6, Bertoldi et al. 2003b). As in the other 
sources, the detection of $\mathrm{CO}(7 \rightarrow 6)$ emission in $\mathrm{J} 1409+5628$ indicates the presence of warm and dense molecular gas.

Assuming a constant brightness temperature from $\mathrm{CO}(3 \rightarrow 2)$ to $\mathrm{CO}(1 \rightarrow 0)$ and a conversion factor from $L_{\mathrm{CO}(1 \rightarrow 0)}^{\prime}$ to molecular mass of $X_{\mathrm{CO}} \approx 0.8 M_{\odot}\left(\mathrm{K} \mathrm{km} \mathrm{s}^{-1} \mathrm{pc}^{2}\right)^{-1}$, appropriate for ultraluminous galaxies (Downes \& Solomon $1998)$, the $\mathrm{CO}(3 \rightarrow 2)$ luminosity implies a mass of molecular gas of $M\left(\mathrm{H}_{2}\right) \approx 6.6 \times 10^{10} M_{\odot} . \mathrm{J} 1409+5628$ appears therefore to be a system which is rich in molecular gas and comparable to the other high- $z$ quasars detected in CO.

\subsection{Radio continuum emission}

Assuming a low frequency spectral index of -0.8 , the $1.4 \mathrm{GHz}$ continuum flux density implies a rest frame luminosity density at $1.4 \mathrm{GHz}$ of $4.0 \times 10^{25} \mathrm{~W} \mathrm{~Hz}^{-1}$. Using the far-infrared luminosity definition of Condon (1992), extrapolated from the $250 \mathrm{GHz}$ flux density of Omont et al. (2003), a dust temperature of $45 \mathrm{~K}$ and a spectral index $\beta=1.5$, the radio-to-FIR ratio is then $q=2.01$, where $q$ is defined by Condon (1992). A tight and linear correlation between radio and far-infrared luminosity has been found in star forming galaxies, with small scatter over a few orders of magnitude in luminosity (Condon 1992). The most recent consideration of this correlation found a value of $q=2.3 \pm 0.3$ for star forming galaxies from the IRAS $2 \mathrm{Jy}$ sample (Yun et al. 2001). Hence, the value of $q$ for J1409+5628 falls well within the envelope defined by star forming galaxies.

Condon et al. (1991) derived an empirical upper limit to the brightness temperature for nuclear starbursts of order $10^{5} \mathrm{~K}$ at $8 \mathrm{GHz}$, while typical radio loud AGN have brightness temperatures two or more orders of magnitude larger than this value. They also present a possible physical model for this limit involving a mixed non-thermal and thermal radio emitting (and absorbing) plasma, constrained by the radio-to-FIR correlation for star forming galaxies. For J1409+5628, we use the observed surface brightness limit from the VLBA together with the measured spectral index and redshift to derive a $4 \sigma$ upper limit to the intrinsic brightness temperature of $2 \times 10^{5} \mathrm{~K}$ at $8 \mathrm{GHz}$ typical of starburst galaxies. This is in contrast with the results obtained by Momjian et al. (2004) for a sample of three high- $z$ quasars which were imaged with the VLBA. In these $z>4$ quasars, the radio-loud AGN dominates the radio emission on very compact size, i.e. a few milliarcsec, with intrinsic brightness temperatures in excess of $10^{9} \mathrm{~K}$.

\subsection{Starburst activity}

Overall, the properties of the $\mathrm{CO}$ emission and the radio emission from $\mathrm{J} 1409+5628$ are consistent with those expected for a star forming galaxy. The radio emission indicates a $q$ value which is consistent with the radio-to-FIR correlation of star forming galaxies, and an intrinsic brightness temperature below $2 \times 10^{5} \mathrm{~K}$ at $8 \mathrm{GHz}$ typical of nuclear starbursts.

The ratio between the far-infrared and $\mathrm{CO}(1 \rightarrow 0)$ luminosities of $\mathrm{J} 1409+5628$ implied by the present observations, $L_{\mathrm{FIR}} / L_{\mathrm{CO}(1 \rightarrow 0)}^{\prime} \approx 500 L_{\odot}\left(\mathrm{K} \mathrm{km} \mathrm{s}^{-1} \mathrm{pc}^{2}\right)^{-1}$ or $L_{\mathrm{FIR}} / L_{\mathrm{CO}(1 \rightarrow 0)} \approx$ $1.1 \times 10^{7}$ is at the upper end of the values derived for local ultraluminous infrared galaxies by Solomon et al. (1997), and in agreement with other high- $z$ quasars where $\mathrm{CO}$ line emission is detected (Cox et al. 2002).

Following Omont et al. (2001), the star formation rate (SFR) in J1409+5628 can be derived from its far-infrared luminosity, assuming that star formation is the main contribution to the dust heating. Depending on the stellar initial mass function and the starburst age and duration, the ratio of SFR to infrared luminosity is in the range 0.8 to $2 \times 10^{-10} M_{\odot} \mathrm{yr}^{-1} / L_{\odot}$, yielding for $\mathrm{J} 1409+5628$ a SFR in between 3 and $8 \times 10^{3} M_{\odot} \mathrm{yr}^{-1}$. The massive reservoir of molecular gas of $6 \times 10^{10} M_{\odot}$ can therefore fuel star formation at the rate implied by the far-infrared luminosity for about 10 million years, a typical duration for a starburst in local galaxies. The dense warm molecular gas is thus rapidly consumed unless its mass only represents a fraction of the total gas available in the host galaxy of $\mathrm{J} 1409+5628$ perhaps in a halo, like in the case of M 82 (Walter et al. 2002). Infalling gas could then further sustain the starburst activity in $\mathrm{J} 1409+5628$ over longer periods of time.

Multiple structures such as double source, arc or ring, which are expected for gravitational lensing, are not detected in the maps of the non-thermal radio continuum on any scale from 10's of mas to a few arcsec. From the radio maps, there is therefore no evidence for lensing in $\mathrm{J} 1409+5628$. No correction for magnification has therefore been applied in this paper. Deep optical and/or near-infrared observations would be useful to further search for evidence of lensing in J1409+5628.

The VLBA observations, which resolve out structures larger than $0{ }^{\prime} 15$, indicate that the $1.4 \mathrm{GHz}$ radio continuum emission should be distributed on scales larger than $1 \mathrm{kpc}$. On the other hand, as shown by the CO observations, the molecular gas is distributed in a region smaller than $5 \mathrm{kpc}$. If the radio continuum and CO emission are co-spatial, the present limits could be compatible with a scenario wherein the starburst activity in $\mathrm{J} 1409+5628$ is confined within a molecular torus or disk of size between 1 and $5 \mathrm{kpc}$. Similar structures with sizes of typically a few kpc are found in the cases of PSS 2322+1944 (Carilli et al. 2003) and the Cloverleaf (Venturini \& Solomon 2003).

Using the limits to the size derived from the $\mathrm{CO}$ and radio observations and assuming that the molecular gas is distributed in a disk, the dynamical mass is $\left(3<M_{\text {dyn }}<10\right) \times$ $10^{10} \sin ^{-2} i M_{\odot}$, where $i$ is the inclination to the line of sight. This range is comparable to the dynamical masses derived for other high- $z$ quasars and galaxies and to that of local $m^{*}$-galaxies (Genzel et al. 2003). The ratio of molecular to dynamical mass is therefore estimated to be $\left(2.4>M_{\mathrm{H}_{2}} / M_{\text {dyn }}>\right.$ 0.6) $\sin ^{2} i$, indicating that a large fraction of the total mass is in the form of molecular gas.

\section{Conclusions}

The millimetre and radio observations described in this paper provide strong evidence that the far-infrared luminosity of $\mathrm{J} 1409+5628$ is related to massive star formation. First, the huge reservoir of dense and warm molecular gas $\left(6 \times 10^{10} M_{\odot}\right)$ can sustain the star formation rate of a few thousand solar masses per year implied by the far-infrared luminosity for 
about 10 million years. Second, the radio continuum emission is found to be consistent with the radio/far-IR correlation of star forming galaxies. The limits on the size of the $\mathrm{CO}$ and radio continuum emitting region suggest that in J1409+5628 star formation is taking place in an extended region, which could be a torus or disk-like structure not larger than $5 \mathrm{kpc}$. Observations of the $\mathrm{CO}$ emission at higher spatial resolution would be useful to further constrain the size of the star-forming region in $\mathrm{J} 1409+5628$ and thereby its dynamical mass.

The submillimeter and radio properties of the $z=$ 2.58 quasar $\mathrm{J} 1409+5628$, which are similar to those seen in other high- $z$ optically luminous quasars, are consistent with a coeval starburst and AGN. However, the relative importance of the warm dust heated by the star formation and the dust directly heated by the AGN is still poorly constrained. Observations at higher frequencies are needed to analyze the rest-frame nearto mid-infrared spectral energy distribution of distant quasars like J1409+5628. Such observations will allow us to derive the total infrared luminosity of high- $z$ quasars, to estimate the contribution of the hot dust heated by the AGN, to better constrain the star formation history in their associated host galaxies and, thereby, to investigate further the coeval growth of stars and massive black holes and its evolution over cosmic time.

Acknowledgements. We thank the IRAM Plateau de Bure staff for their support, V. T. Junkkarinen for sending us information on the optical Keck spectrum of J1409+5628 before publication and A. Weiss for helpful comments. We also thank an anonymous referee for comments which improved the content of this paper.

\section{References}

Barkhouse, W. A., \& Hall, P. B. 2001, AJ, 121, 2843

Barlow, T. A., \& Junkkarinen, V. T. 1994, Bull. Am. Astron. Soc., 26, 1339

Barvainis, R., Maloney, P., Antonucci, R., \& Alloin, D. 1997, ApJ, 484, 695

Bertoldi, F., Carilli, C. L., Cox, P., et al. 2003a, A\&A, 406, L55

Bertoldi, F., Cox, P., Neri, R., et al. 2003b, A\&A, 409, L47
Blain, A. W., Smail, I., Ivison, R. J., Kneib, J.-P., \& Frayer, D. T. 2002 , Phys. Rep., 369, 111

Carilli, C. L., Lewis, G. F., Djorgovski, S. G., et al. 2003, Science, 300,773

Carilli, C. L., Bertoldi, F., Walter, F., et al. 2004, in the Proc. of Conf. on multiwavelength AGN surveys, Cozumel, Mexico, 8-12 Dec. 2003, in press

Chapman, S. C., Blain, A. W., Ivison, R. J., \& Smail, I. R. 2003, Nature, 422, 695

Condon, J. J. 1992, ARA\&A, 30, 575

Condon, J. J., Huang, Z.-P., Yin, Q. F., \& Thuan, T. X. 1991, ApJ, 378, 65

Cox, P., Omont, A., Djorgovski, S. G., et al. 2002, A\&A, 387, 406

Downes, D., \& Solomon, P. M. 1998, ApJ, 507, 615

Downes, D., \& Solomon, P. M. 2003, ApJ, 582, 37

Fan, X., Strauss, M. A., Schneider, D. P., et al. 2003, AJ, 125, 1649

Fomalont, E. 1999, in Synthesis imaging in radio astronomy II, ASP Conf. Ser., 180, 301

Genzel, R., Baker, A. J., Tacconi, L. J., et al. 2003, ApJ, 584, 633

Ivison, R. J., Smail, I., Barger, A. J., et al. 2000, MNRAS, 315, 209

Korista, K. T., Voit, G. M., Morris, S. L., \& Weymann, R. J. 1993 , ApJS, 88, 357

Momjian, E., Petric, A. O., \& Carilli, C. L. 2004, AJ, 127, 587

Neri, R., Genzel, R., Ivison, R. J., et al. 2003, ApJ, 597, L113

Omont, A., Cox, P., Bertoldi, F., et al. 2001, A\&A, 374, 371

Omont, A., Beelen, A., Bertoldi, F., et al. 2003, A\&A, 398, 857

Petitjean, P., Rauch, M., \& Carswell, R. F. 1994, A\&A, 291, 29

Priddey, R. S., Isaak, K. G., McMahon, R. G., Robson, E. I., \& Pearson, C. P. 2003, MNRAS, 344, L74

Richards, G. T., Vanden Berk, D. E., Reichard, T. A., et al. 2002, AJ, 124,1

Shaver, P. A., Wall, J. V., Kellermann, K. I., Jackson, C. A., \& Hawkins, M. R. S. 1996, Nature, 384, 439

Solomon, P., Vanden Bout, P., Carilli, C., \& Guelin, M. 2003, Nature, 426, 636

Solomon, P. M., Downes, D., Radford, S. J. E., \& Barrett, J. W. 1997, ApJ, 478, 144

Spergel, D. N., Verde, L., Peiris, H. V., et al. 2003, ApJS, 148, 175

Venturini, S., \& Solomon, P. M. 2003, ApJ, 590, 740

Walter, F., Weiss, A., \& Scoville, N. 2002, ApJ, 580, L21

Walter, F., Bertoldi, F., Carilli, C., et al. 2003, Nature, 424, 406

Yun, M. S., Reddy, N. A., \& Condon, J. J. 2001, ApJ, 554, 803 